\title{
Evaluation of mechanical properties of berries on resistant or tolerant varieties of grapevine
}

\author{
Duilio Porro,", Marco Wolf, and Stefano Pedò \\ Technology Transfer Centre, Edmund Mach Foundation, via E. Mach 1, 38010 San Michele all'Adige, Italy
}

\begin{abstract}
An experimental site located in Trentino (North-Eastern Italy), characterized by considerable rainfall that normally requires several plant protection treatments, was used to assess the behavior of 15 grape varieties resistant to the main fungal diseases from an agronomic, quantitative, qualitative, nutritional, and physiological point of view, since 2015. At the 4 th year of planting (2016), mechanical properties (berry firmness, berry skin hardness, and thickness) of berries were evaluated using a TAxT2i Texture Analyzer in order to get information about parameters useful for wine process. The varieties showed significant differences in the studied parameters. Regent had the highest values of berry firmness, whilst Johanniter and Cabino the lowest. Cabernet Cantor and Cabernet Cortis generally presented higher values of berry skin hardness than the other varieties. Conversely, the lowest values of skin hardness were recorded in Johanniter, followed by both Solaris and Cabino. Souvignier gris, Prior, and Bronner had significantly higher values of berry skin thickness than Cabino, which, in turn, did not differ from the levels found in Helios, Muscaris, Aromera, and Regent berries, with values above $175 \mu \mathrm{m}$, range largely found in grapevine cultivars normally grown. The association between mechanical properties of berries and qualitative data could be used as an aid in decision-making about wine processes.
\end{abstract}

\section{Introduction}

In the last few years, one of the most important goals of viticulture has been the selection grapevine cultivars less demanding in terms of pest protection, to be used as a valid alternative to the main cultivated varieties of Vitis vinifera. In fact, the main cultivars normally require a large number of treatments to fight both downy mildew (Plasmopara viticola) and powdery mildew (Uncinula necator and Oidium tuckeri) during the growing season. The meteorological conditions in Trentino are characterized by rainfall that exceeds an average of 800 $\mathrm{mm}$ per year, requiring several treatments aimed at providing phytosanitary defense.

For this reason, therefore, the identification of more resistant and/or tolerant cultivars, mainly to downy mildew, can be an interesting strategy for a more sustainable viticulture.

Some resistant and/or tolerant varieties recently identified - resulting from interspecific cross between cultivars of Vitis vinifera and other Vitis of American and Asian origin - have been under observation for some years in the Trentino viticultural area. The main agronomic, physiological, and nutritional aspects of these genotypes were evaluated $[1,2]$.

Of these aspects, some data are available, but they need to be validated with greater repeatability in the coming years. The evaluation of these varieties for a better placement at an enological and market level, however, is currently still lacking and requires further trials aimed to refine the techniques of production of specific wines.

This preliminary work has been carried out in order to obtain information on this topic, in particular analyzing the appearance of the mechanical properties of berries (berry firmness, skin hardness and skin thickness), referring to qualitative parameters too. This was done to support decision-making for the identification of optimal technological solutions for wine processes able to enhance the grapes coming from these resistant and/or tolerant varieties.

\section{Materials and methods}

In 2013, fifteen different genotypes of grapevine resistant/tolerant to the main fungal diseases, such as downy mildew and powdery mildew, were planted in Vallagarina (Trentino, North-Eastern Italy). The experimental site is located in Rovereto-Navicello (lat:: $46^{\circ} 52^{\prime} 37.96$ "N, long.: $11^{\circ} 01^{\prime} 14.03 " \mathrm{E}$ ), with an altitude of $220 \mathrm{~m}$ above sea level, on a sandy, subalkaline, extremely calcareous soil, characterized by good level of active limestone (3.8\%) and organic matter $(2.3 \%)$. The soil presents a high availability of phosphorus and magnesium, a medium content in potassium, and a high $\mathrm{Mg} / \mathrm{K}$ ratio.

\footnotetext{
Corresponding author: duilio.porro@,fmach.it
} 
The resistant cultivars tested - Aromera, Baron, Bronner, Cabernet Cantor, Cabernet Carbon, Cabernet Cortis, Cabino, Helios, Johanniter, Monarch, Muscaris, Prior, Regent, Solaris, and Souvignier gris - are present in a varietal collection composed of about 100 plants of each genotype, trained to pergola Trentina system with a plant density of 4329 vines/hectare $(3 \mathrm{~m} \times 0.77 \mathrm{~m})$. No antifungal treatments were performed during each growing season.

Starting from the third year, nutritional aspects were monitored at the phenological phase of fruit set by using leaf analyses, as well as physiological aspects by detecting the intensity of leaf greenness (SPAD values) and the photosynthetically active biomass (NDVI index). Every year, at harvest time, quantitative (yield) and qualitative $\left({ }^{\circ}\right.$ Brix, titratable acidity -TA, $\mathrm{pH}$, malic acid, tartaric acid, potassium) parameters of musts were collected. Nevertheless, in order to obtain different wines, grapes were harvested annually and processed into microvinifications. The obtained wines were then tasted during the springtime of each following year. With regard to these data, it should be noted that in the present work they are not reported, as they are partially present in another work [2].

In 2016, four years after planting, from each cultivar under observation, mechanical properties of the berries (berry firmness, skin hardness and skin thickness), also called rheological parameters, were evaluated by puncture and compression test [3, 4], using a Universal Testing Machine TAxT2i Texture Analyzer (Stable Micro System, Godalming, Surrey, UK). For the evaluation of berry skin thickness, before Texture Analyzer measurements, samples of berries were frozen with liquid nitrogen [5]. For each mechanical parameter, a sample of 30 berries was used.

Quantitative (average yield/hectare) and qualitative $\left({ }^{\circ} \mathrm{Brix}, \mathrm{pH}\right.$ and TA) data of each cultivar recorded in 2016 and obtained from the average of 14 plants per genotype, are used in this work to highlight potential enological products.

All data were statistically processed using Systat software package (Systat Software Inc., USA), separating the averages by Tukey test. In the text, the values statistically different are indicated by different letters. The levels of significance reported and indicated with n.s., *, **, ***, represent respectively not significant, significance for values of $\mathrm{P} \leq 0.05$ and $\mathrm{P} \geq$ $0.01, \mathrm{P}<0.01$ and $\mathrm{P} \geq 0.001, \mathrm{P}<0.001$. The multivariate procedure of Cluster Analysis was applied to the mean values of parameters related to mechanical properties of the berries. Normalized Euclidean distances (root meansquared distances) were used.

\section{Results and discussion}

The investigated varieties showed significant differences for the rheological parameters studied (table 1).

Regent had significantly higher values of berry firmness than all the other cultivars. The lowest values of this rheological parameter were found in Johanniter and Cabino.
The skin hardness test showed that both Cabernet Carbon and Cabernet Cortis cultivars generally had higher values than the other varieties. The lower values of this parameter, however, were recorded on the berries of Johanniter, followed by those of Solaris and Cabino. Regarding skin thickness, Souvignier gris, Prior, and Bronner showed the highest values, whilst Cabino the lowest ones, although this cultivar did not statistically differ from Helios, Muscaris, Aromera, Johanniter, and Regent.

Table 1: mean values of parameters related to mechanical properties of berries (berry firmness, skin hardness, and skin thickness) in relation to cultivar. For each parameter, 450 cases

\begin{tabular}{|c|c|c|c|}
\hline \multirow[b]{2}{*}{ Variety } & \multicolumn{3}{|c|}{ Parameter } \\
\hline & $\begin{array}{c}\text { Berry } \\
\text { firmness }(\mathrm{g})\end{array}$ & $\begin{array}{c}\text { Skin } \\
\text { hardness } \\
\text { (Newton) }\end{array}$ & $\begin{array}{l}\text { Skin } \\
\text { thickness } \\
\quad(\mu \mathrm{m})\end{array}$ \\
\hline Aromera & $622 \mathrm{de}$ & $0.406 \mathrm{def}$ & $165 \mathrm{fg}$ \\
\hline Baron & $597 \mathrm{def}$ & $0.451 \mathrm{bcde}$ & 187 bcde \\
\hline Bronner & 572 ef & $0.423 \mathrm{cdef}$ & $204 \mathrm{ab}$ \\
\hline C. Cantor & $917 \mathrm{~b}$ & $0.519 a b$ & $181 \mathrm{cdef}$ \\
\hline C. Carbon & $765 \mathrm{bcd}$ & $0.591 \mathrm{a}$ & 189 bcde \\
\hline C. Cortis & $836 \mathrm{bc}$ & $0.596 \mathrm{a}$ & $192 \mathrm{bcd}$ \\
\hline Cabino & $423 \mathrm{f}$ & $0.366 \mathrm{fg}$ & $157 \mathrm{~g}$ \\
\hline Helios & $681 \mathrm{cde}$ & $0.429 \mathrm{cdef}$ & $165 \mathrm{fg}$ \\
\hline Johanniter & $413 \mathrm{f}$ & $0.320 \mathrm{~g}$ & 173 defg \\
\hline Monarch & 704 cde & 0.381 efg & 189 bcde \\
\hline Muscaris & 739 bcde & $0.473 \mathrm{bcd}$ & $161 \mathrm{fg}$ \\
\hline Prior & 733 bcde & $0.425 \mathrm{cdef}$ & $202 a b c$ \\
\hline Regent & $1217 \mathrm{a}$ & $0.494 \mathrm{bc}$ & 167 efg \\
\hline Solaris & 702 cde & $0.367 \mathrm{fg}$ & $193 \mathrm{bcd}$ \\
\hline Souvignier $g$. & 756 bcde & $0.484 \mathrm{bcd}$ & $216 \mathrm{a}$ \\
\hline Significance & $* * *$ & $* * *$ & $* * *$ \\
\hline
\end{tabular}

It is interesting to remark that these latter varieties presented lower levels than the average normally recorded for cultivated grapevine of Vitis vinifera, which generally ranged around values of $175 \mu \mathrm{m}$ [6].

The average values of berry firmness, skin hardness and skin thickness of Pinot gris and Cabernet franc (data not shown in table 1), varieties particularly widespread at international level and taken as a reference, were recorded in the same year of the trial. The values were respectively $530 \mathrm{~g}, 0.580$ Newton and $172.5 \mu \mathrm{m}$ in the white berry cultivar and $1047 \mathrm{~g}, 0.733$ Newton and 143.6 $\mu \mathrm{m}$ in the red berry cultivar.

It should be noted that Cabernet franc has been chosen because it represents one of the cultivars characterized by the lowest skin thickness values (below $150 \mu \mathrm{m}$ ) compared to those of the main cultivated grapevines [7]. The comparison of the three rheological parameters of the studied cultivars with these reference varieties was made using the Cluster Analysis. The results reported in figure 1 allow identification of the positioning of 
resistant and/or tolerant genotypes tested with respect to those identified as an international reference. From a technological point of view, these results may be used as an aid in decision-making about wine processes.

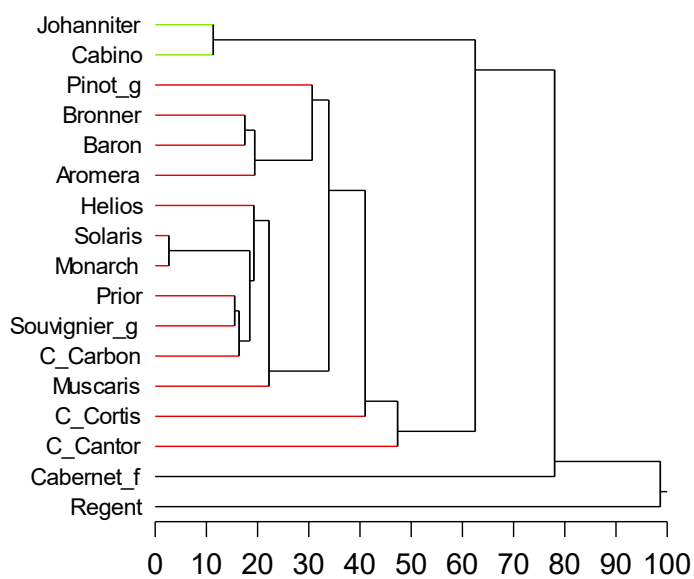

Fig. 1: grouping of the cultivars in relation to the three mechanical properties of the berries through Cluster Analysis. Normalized Euclidean distances are reported.

The separation of the different groups of cultivars places to the extreme limits of the Cluster, Johanniter, and Cabino on one hand with low skin hardness values and medium-low thickness, and on the other hand Regent and Cabernet franc, with high levels of berry firmness and low skin thickness values.

The cultivars Baron, Bronner, and Aromera were found very close to Pinot gris; they were indeed characterized by medium-low values of both berry firmness and skin hardness, but with different thickness levels compared to those recorded in Pinot gris, with respectively low values in Aromera, medium-high in Baron and rather high in Bronner.

Solaris and Monarch, characterized by medium-low values of berry firmness and skin hardness, were grouped with Helios. The first two recorded an average level of skin thickness, different from the very low levels observed in the latter.

The group composed by Muscaris, Cabernet Carbon, Souvignier gris, and Prior has been unified for mediumhigh values of berry firmness and skin hardness, but with very different values of skin thickness, ranging from low levels (Muscaris and Cabernet Carbon) to very high ones (Souvignier gris and Prior).

Finally, Cabernet Cantor and Cabernet Cortis have been placed close to the varieties characterized by high levels of berry firmness and skin hardness, but with low skin thickness values.

Average values of quantitative (yield/hectare) and qualitative data of both resistant cultivars and varieties taken as a reference recorded in 2016 are reported in table 2. Aromera, Bronner, Cabernet Cantor, Cabernet Cortis, Johanniter, Monarch, Muscaris, Solaris, and Souvignier gris showed interesting production per hectare, ranging from 7.5 to 14 tonnes. Both Pinot gris and Cabernet franc presented yield around 15 tonnes.
Baron, Cabernet Carbon, Cabino, Helios, Prior, and Regent had very low production.

Table 2: mean values of quantitative and qualitative parameters in relation to cultivar.

\begin{tabular}{|l|c|c|c|c|}
\hline \multirow{2}{*}{ Variety } & \multicolumn{3}{|c|}{ Parameter } \\
\cline { 2 - 5 } & $\begin{array}{c}\text { Yield } \\
(\mathrm{t} / \text { hectare })\end{array}$ & o Brix & $\mathrm{pH}$ & $\begin{array}{c}\text { TA } \\
(\mathrm{g} / \mathrm{L})\end{array}$ \\
\hline Aromera & 9.5 & 18.59 & 3.29 & 5.4 \\
\hline Baron & 1.9 & 21.99 & 3.54 & 4.2 \\
\hline Bronner & 12.7 & 20.19 & 3.21 & 7.1 \\
\hline C. Cantor & 7.8 & 25.43 & 3.19 & 6.9 \\
\hline C. Carbon & 5.7 & 21.24 & 3.21 & 7.0 \\
\hline C. Cortis & 8.0 & 23.45 & 3.08 & 6.5 \\
\hline Cabernet franc & 15.0 & 23.16 & 3.49 & 3.2 \\
\hline Cabino & 3.1 & 18.18 & 3.51 & 6.5 \\
\hline Helios & 3.1 & 20.81 & 3.33 & 5.6 \\
\hline Johanniter & 13.6 & 17.85 & 3.55 & 5.2 \\
\hline Monarch & 13.2 & 16.51 & 3.11 & 8.0 \\
\hline Muscaris & 12.1 & 24.17 & 3.35 & 7.3 \\
\hline Pinot gris & 14.9 & 22.28 & 3.53 & 5.3 \\
\hline Prior & 4.8 & 20.40 & 3.31 & 7.1 \\
\hline Regent & 4.1 & 20.98 & 3.77 & 6.2 \\
\hline Solaris & 13.7 & 25.10 & 3.24 & 7.2 \\
\hline Souvignier g. & 9.3 & 23.49 & 3.39 & 7.4 \\
\hline
\end{tabular}

Cabernet Cantor, Cabernet Cortis, Muscaris, Solaris, and Souvignier gris presented the highest sugar levels, whilst Aromera, Cabino, Johanniter, Prior, and Monarch the lowest ones. Cabernet Cortis and Monarch showed the lowest values of $\mathrm{pH}$, conversely to Regent which had the highest. Monarch and Baron had respectively the highest and lowest levels of acidity.

The association of data on mechanical properties with those of production (yield) and quality of musts did not show any important correlations able to provide useful technological suggestions for the enhancement of the different resistant varieties from an enological point of view. However, although with low level of $\mathrm{r}$ square, values of skin hardness were positively correlated with sugar levels $\left(\mathrm{r}^{2}=0.21\right)$, as well as those of skin thickness with acidity $\left(r^{2}=0.30\right)$.

Some rheological parameters, in particular, skin hardness and thickness, as recently reported [8], appear to be related to the quantity of extractable anthocyanins and to the amount of thiolic precursors, thus intervening both on the wine coloring and on the aromatic nature of the same. Due to these links, it clearly emerges that the evaluation of mechanical properties of berries and their association with qualitative and technological parameters need to be further investigated in order to finalize optimal winemaking processes specific for the resistant varieties. In fact, some of these cultivars characterized 
by high levels of both skin thickness and hardness can be left for a long time in maceration in order to increase the concentration of thiol precursors [9]. In fact, as recently reported [10], some grape skin tannins were shown to contain very high amounts of 3-S-glutathionylhexan-1-ol and 3-S-cysteinylhexan-1-ol (polyfunctional thiol precursors), whose free forms are responsible for appreciated tropical-like flavours.

\section{Conclusions}

Data collected about rheological properties of the berries of resistant and/or tolerant cultivars, albeit limited to a single year, can undoubtedly constitute a source of fundamental information.

Similar measurements must be further validated by repeating them over several years and, moreover, by relating them to qualitative and productive data in order to properly finalize the winemaking processes.

If associated with the information deriving from the degree of fungal attack and/or entomological diseases, skin thickness data could be extremely important for a specifically targeted management of the different cultivars under observation.

\section{References}

1. D. Porro, S. Pedò, M. Bottura. Acta Italus Hortus, 19, 131-132 (2016).

2. S. Pedò, M. Bottura, D. Porro. BIO web of conferences (to be published).

3. H. Letaief, L. Rolle, V. Gerbi. Am. J. Enol. Vitic., 59 (3), 323-329 (2008).

4. H. Letaief, L. Rolle, G. Zeppa, V. Gerbi. J Sci Food Agric, 88, 1567-1575 (2008).

5. D. Porro, T. Tomasi, M. Ramponi, L. Rolle, S. Poni. Acta Hort., 868, 73-80 (2010).

6. D. Porro, S. Ferrarin, P.L. Bianchedi, S. Clementi, A. Vecchione, M. Stefanini M. Annual report IASMA Research Centre, 36-38 (2009).

7. F. Battista, D. Tomasi, D. Porro, F. Caicci, S. Giacosa, L. Rolle. Ital. J. Food Sci., 27, 136-141 (2015).

8. L. Rolle, F. Torchio, G. Zeppa, V. Gerbi. Am. J. Enol. Vitic., 60 (1), 93-97 (2009).

9. T. Román, L. Tonidandel, R. Larcher, E. Celotti, G. Nicolini. Eur Food Res Technol, 244, 379-386 (2018).

10. T. Román Villegas, L. Tonidandel, B. Fedrizzi, R. Larcher, G. Nicolini. Food Chem, 207, 16-19 (2016). 